\title{
Trimodality bladder-sparing approach without neoadjuvant chemotherapy for node-negative localized muscle-invasive urinary bladder cancer resulted in comparable cystectomy-free survival
}

Cheng-Yen Lee ${ }^{1 \dagger}$, Kai-Lin Yang ${ }^{1,4+}$, Hui-Ling Ko ${ }^{1}$, Rong-Yau Huang ${ }^{3}$, Pei-Pin Tsai ${ }^{3}$, Ming-Tsun Chen ${ }^{3}$, Yi-Chia Lin ${ }^{2,4}$, Thomas I-Sheng Hwang ${ }^{2,4}$, Guang-Dar Juang ${ }^{2,4^{*}}$ and Kwan-Hwa Chi ${ }^{1,5^{*}}$

\begin{abstract}
Background: To retrospectively review the efficacy and organ preservation experience for muscle-invasive bladder cancer by trimodality therapy at our institution.

Methods: Between July 2004 and February 2012, seventy patients (M/F $=55 / 15$; median age $=69$ years) of lymph node negative localized muscle-invasive bladder cancer were treated primarily with trimodality approach including transurethral resection of bladder tumor (TURBT) prior to combined chemotherapy and radiotherapy (CCRT). Radiotherapy consisted of initial large field size irradiation with 3D conformal technique (3D-CRT), followed by cone-down tumor bed boost with intensity modulated radiotherapy (IMRT) technique. The median total doses delivered to bladder tumor bed and whole bladder were 59.4Gy and 40.0Gy, respectively. No patient received neoadjuvant chemotherapy (NAC). Weekly cisplatin was administered during radiotherapy. Toxicity was scored according to the RTOG criteria. Tumor response was evaluated both cystoscopically and radiographically 3 months after treatment.

Results: The numbers of patients with T2, T3 and T4 lesions were 41, 16 and 13, respectively. Overall survival (OS) and progression-free survival (PFS) at 2 and 5 year were $65.7 \%, 51.9 \%$ and 50.8\%, 39.9\%, respectively, after a median follow-up time of 24 months. Local-regional control and distant metastasis free survival at 2 year were $69.8 \%$ and $73.5 \%$, respectively. Complete response (CR) rate assessed three month after CCRT was $78.1 \%$. Ten patients (20\%) had local recurrence after initial $C R(n=50), 3$ of them were superficial recurrence. One patient underwent radical cystectomy after recurrence. The overall 5-year bladder intact survival was $49.0 \%(95 \% \mathrm{Cl}$, $35.5 \%$ to $62.5 \%$ ). Acute toxicities were limited to grade 1-2. One patient developed late grade $3 \mathrm{GU}$ toxicity. Conclusions: Our result suggested that trimodality bladder-sparing approach without NAC or dose-intensification could be well-tolerated with a high CR rate and bladder preserving rate for muscle-invasive bladder cancer.
\end{abstract}

Keywords: Urinary bladder cancer, Chemoradiation, Trimodality, Organ preservation

\footnotetext{
*Correspondence: M000733@ms.skh.org.tw; M006565@ms.skh.org.tw

${ }^{\dagger}$ Equal contributors

${ }^{2}$ Division of Urology, Department of Surgery, Shin Kong Wu Ho-Su Memorial Hospital, No. 95, Wen-Chang Road, Shih-Lin District, Taipei City, Taiwan

'Department of Radiation Therapy and Oncology, Shin Kong Wu Ho-Su Memorial Hospital, No. 95, Wen-Chang Road, Shih-Lin District, Taipei City, Taiwan

Full list of author information is available at the end of the article
} 


\section{Background}

Historically, surgery was the main treatment of muscleinvasive bladder cancer. Radical cystectomy with urinary diversion and pelvic lymph node dissection resulted in 5 -year pelvic control rate of $80-90 \%$ and 5-year OS of $40-60 \%$ [1]. Radical cystectomy and partial cystectomy (in selected cases) are frequently regarded as standard of care in contemporary management of muscle-invasive bladder cancer, where bladder-sparing radiotherapy was used as an alternative. Compared with radiotherapy alone, an OS benefit was observed with radical surgery in patients with muscle-invasive bladder cancer [2]. The introduction of bladder sparing therapy with or without NAC besides CCRT had been pioneered by Tester et al [3], Shipley et al [4] and Rodel et al [5]. CCRT has soon be regarded as the mainstream of organ preservation therapy instead of RT alone [6]. Although intriguing, the superiority of NAC has not been proven in randomized trial [4]. However, most trimodality treatment consisted of NAC followed by CCRT, with radical cystectomy reserved for incomplete responders in these reports [7]. Overall, the 5 -year OS was approximately $50 \%$ with three-quarters of them maintaining functional bladders [7]. A trend toward increasing the intensity of chemotherapy during CCRT, such as the addition of vinblastine, paclitaxel and gemcitabine to cisplatin [8-11], and escalation of biological radiation dosage via hyperfractionation and hypofractionation was noted $[9,12]$. However, late pelvic toxicity are concerning. In spite of these encouraging results and possible positive impact on quality of life [13], widespread use of this technique was still limited. In a report by Fedeli et al, the majority of muscle invasive bladder cancer in the United States were still treated with cystectomy $(42.9 \%)$, while the minority were treated radiation therapy (16.6\%) [14].

We hereby report our institutional experience on bladder preservation using upfront CCRT without NAC or adjuvant chemotherapy, with IMRT boost after whole bladder irradiation by 3D-CRT.

\section{Methods}

\section{Patient characteristics}

Between July 2004 and February 2012, 91 patients diagnosed with muscle-invasive (T2 to T4) bladder cancer who were treated with curative intent in our institution were retrospectively reviewed. Patients with pelvic lymph node metastases and distant metastases, as detected by computed tomography (CT), magnetic resonance imaging (MRI) or other modalities before RT were excluded in this review. Seventy patients with newly diagnosed or recurrent disease after initial TURBT treated with CCRT after TURBT or partial cystectomy were included in this analysis. This review was approved by the institutional review board of our hospital (No.20130506R). T-category was re-assigned according to the American Joint Committee on Cancer (AJCC) tumor-node-metastasis classification of 2010. The majority of patients included in the study had pathological evidence of tumoral invasion of the muscular layer, either after a TURBT $(\mathrm{n}=64 ; 91.4 \%)$ or partial cystectomy $(\mathrm{n}=4,5.7 \%)$. The remaining patients $(\mathrm{n}=2 ; 2.9 \%)$ received only biopsy, but had radiographical evidence of T4 disease. The majority of the population had urothelial cell carcinoma $(n=65,92.9 \%) .55$ of the patients $(78.6 \%)$ had high grade disease on pathology.

\section{Treatment}

Patients were referred from urologist. Those patients were not candidates for radical cystectomy because of comorbidities or personal preference. Radiotherapy was performed 4 to 8 weeks after TURBT or partial cystectomy. Planning CT scan with 3-mm slices was acquired for all patients undergoing RT. The patients were simulated in supine position with distended bladder. They were instructed to void and take a fixed amount of fluid $(350-500 \mathrm{~mL})$ thirty minutes prior to both simulation and treatment. Large field irradiation to subclinical disease by 3D-CRT followed by tumor-directed boost by IMRT was our treatment guideline. High risk clinical target volumes $(\mathrm{CTV}-\mathrm{H})$ were defined as preoperative tumor bed, which were delineated on the planning CT with respect to preoperative MRI or CT images registered by image fusion. In those who underwent partial cystectomy, the positions of surgical clips needed to be taken into consideration for delineating CTV-H. In the presence of gross residual tumor, the gross tumor volume (GTV) was delineated on planning CT. The whole bladder was treated as $\mathrm{CTV}-\mathrm{H}$ in the presence of multifocal disease. A $1-2 \mathrm{~cm}$ margin was added to CTV-H and GTV in all directions to account for organ motion and formed the planning target volume (PTV-H). IMRT was delivered via 4-10 MV photons using 5 to 7 static IMRT fields to PTV-H volume. The clinical target volume at intermediate risk (CTV-M) encompassed whole bladder in T2 disease and included pelvic nodal regions (including hypogastric, obturator, external iliac and perivesical nodes) in T3 and T4 disease. A 2-cm margin was given to CTV-M in the cranial and anterior directions and $1-\mathrm{cm}$ in posterior, lateral and caudal directions, forming PTV-M. PTV-M was treated with $10 \mathrm{MV}$ photons by 4-field 3DCRT technique. All treatment planning was performed by Pinnacle version 9.0 (Philips Healthcare, Bothell, WA).

RT was initiated 4 to 8 weeks after urological procedure. Patients were treated with the same requirements as in the planning phase. A median total dose of 59.4Gy (range, 44.5 to 66.6Gy) was delivered to the PTV-H, and the PTV-M was irradiated with a median total dose of 40.0Gy (range, 36.0 to $54.0 \mathrm{~Gy}$ ) at $1.8-2.0$ Gy per daily fraction. 
The prescribed doses should cover at least $95 \%$ of PTV volumes. The dose constraints were $\mathrm{V}_{55}<50 \%$ for rectum, and $\mathrm{D}_{\max }<45 \mathrm{~Gy}$ to both bowels and femoral heads.

Concurrent platinum-based chemotherapy was offered and consisted of cisplatin $\left(30 \mathrm{mg} / \mathrm{m}^{2} /\right.$ week) for patients without impaired renal function defined as glomerular filtration rate $(\mathrm{GFR})>50 \mathrm{~mL} / \mathrm{min}$, or carboplatin

Table 1 Patient, tumor and treatment-related characteristics

\begin{tabular}{|c|c|}
\hline Characteristics & Value \\
\hline Age, years, median (range) & $69(49-92)$ \\
\hline \multicolumn{2}{|l|}{ Gender } \\
\hline Male & $55(78.6 \%)$ \\
\hline Female & $15(21.4)$ \\
\hline \multicolumn{2}{|l|}{ History of prior TUR-BT } \\
\hline Initial treatment & $50(71.4 \%)$ \\
\hline Recurrence after previous TURBT & $20(28.6 \%)$ \\
\hline \multicolumn{2}{|l|}{ ECOG Performance status } \\
\hline 0 & $26(37.1 \%)$ \\
\hline 1 & $43(61.5 \%)$ \\
\hline 2 & $1(1.4 \%)$ \\
\hline \multicolumn{2}{|l|}{ Comorbidities } \\
\hline No & $20(28.6 \%)$ \\
\hline Yes & $50(71.4 \%)$ \\
\hline \multicolumn{2}{|l|}{ Pathology } \\
\hline Transitional cell carcinoma & $65(92.9 \%)$ \\
\hline Adenocarcinoma & $1(1.4 \%)$ \\
\hline Other histology or missing record & $4(5.7 \%)$ \\
\hline \multicolumn{2}{|l|}{ Grade } \\
\hline High & $55(78.6 \%)$ \\
\hline Low & $1(1.4 \%)$ \\
\hline Missing record & $14(20.0 \%)$ \\
\hline \multicolumn{2}{|l|}{ Clinical T stage } \\
\hline $\mathrm{T} 2$ & $41(58.6 \%)$ \\
\hline T3 & $16(22.9 \%)$ \\
\hline T4 & $13(18.6 \%)$ \\
\hline \multicolumn{2}{|l|}{ Focality } \\
\hline Multifocal & $26(37.2 \%)$ \\
\hline Single & $44(62.8 \%)$ \\
\hline \multicolumn{2}{|l|}{ Hydronephrosis } \\
\hline No & $39(55.7 \%)$ \\
\hline Yes & $31(44.3 \%)$ \\
\hline \multicolumn{2}{|l|}{ Procedure before radiotherapy (RT) } \\
\hline Transurethral resection (TUR) & $64(91.4 \%)$ \\
\hline Partial cystectomy & $4(5.7 \%)$ \\
\hline Cystoscopic biopsy only & $2(2.9 \%)$ \\
\hline
\end{tabular}

(100 $\mathrm{mg} / \mathrm{m}^{2}$ on days $\left.1,15,31\right)$ for patients with renal function impairment. The treatment-related characteristics were summarized in Table 1.

\section{Follow-up and evaluation}

Patients were observed at 3-month intervals for the first 3 years and every 6 months thereafter. Post-treatment follow-up consisted of pertinent medical history, physical examination, urine cytology, cystoscopy, and radiological evaluation as clinically indicated. Tumor response was evaluated using the Response Evaluation Criteria in Solid Tumors (RECIST) [15] both cystoscopically and radiographically (either $\mathrm{CT}$ or MRI) three months after treatment. Complete response $(\mathrm{CR})$ was defined as the absence of detectable tumor as well as negative urine cytology. Among patients with less than CR, cystectomy was offered by the treating physicians. Salvage therapy for non-muscle invasive recurrences consisted of TUR-BT followed by intravesical therapy for nonmuscle invasive recurrence and radical cystectomy for muscle-invasive recurrences. However, the final decision was determined at the discretion of urologist and patient's own will. In case of distant failure, combination cisplatin and gemcitabine was offered as first line chemotherapy. Evaluation of late treatment-related toxicity was performed according to the toxicity criteria of the Radiation Therapy Oncology Group (RTOG) and the European Organization for Research and Treatment of Cancer (EORTC) [16,17].

\section{Statistics}

At the time of analysis, the median follow-up for the entire group was 24 months. Survival was measured from the day of start of RT to the date of death or the most recent follow-up visit. The OS and PFS was calculated using the method of Kaplan-Meier and curve comparison by log-rank test. Univariate and multivariate analysis by Cox proportional hazards model were performed to determine clinicopathological factors with prognostic value for OS. A $p$-value $<0.05$ (two-sided) was considered significant in all of the statistical testing. The statistical analysis was performed using Statistical Package for Social Sciences software for Windows version 13.0 (SPSS, Inc, Chicago, IL).

Table 2 Tumor response after radiotherapy

\begin{tabular}{lc}
\hline & Evaluable patients $(\mathbf{n}=\mathbf{6 4})$ \\
\hline Complete response (CR) & $50(78.1 \%)$ \\
Partial response (PR) & $2(3.1 \%)$ \\
Stable disease (SD) & $3(4.7 \%)$ \\
Progressive disease (PD) & $9(14.1 \%)$ \\
\hline
\end{tabular}


Table 3 Clinical outcomes

\begin{tabular}{|c|c|c|}
\hline & 2-year rates & 5-year rates \\
\hline Overall survival & $65.7 \%$ & $50.8 \%$ \\
\hline Bladder cancer specific survival & $77.3 \%$ & $67.3 \%$ \\
\hline Survival with intact bladder ${ }^{*}$ & $64.2 \%$ & $49.0 \%$ \\
\hline Local-regional control & $69.8 \%$ & $61.7 \%$ \\
\hline Distant-metastasis-free survival & $73.5 \%$ & $67.1 \%$ \\
\hline Progression-free survival & $51.9 \%$ & $39.9 \%$ \\
\hline
\end{tabular}

*Only one patient with local failure received radical cystectomy as salvage treatment.

\section{Results}

\section{Tumor response after radiotherapy}

Among the 70 patients, 64 patients were evaluable for response (3 patients died of non-cancer comorbidities shortly after treatment, and 3 patients refused cystoscopic evaluation). Of the evaluable 64 patients, $78.1 \%$ (50 patients) achieved CR (Table 2). Of the remaining patients with non-CR, two (3.1\%) had partial response (PR), three (4.7\%) had stable disease, and nine (11.4\%) had progressive disease. Although radical cystectomy was offered to all patients with less than CR, none of the patients underwent this procedure due to patients' refusal or medical inoperability.

\section{Survival and bladder preservation}

The median survival was 64.6 months after a median follow-up of 24 months. OS and PFS at 2 year were $65.7 \%$ and 51.9\%. Local-regional control and distant metastasis free survival at 2 year were $69.8 \%$ and $73.5 \%$, respectively. Bladder cancer specific survival and survival with intact bladder at 2 year were $77.3 \%$ and $64.2 \%$ respectively. The clinical outcomes at 2 year and 5 year were illustrated in Table 3.

Univariate analysis of 12 treatment-related factors influencing OS was shown in Table 4. Age, performance status, $\mathrm{T}$ stage, prescribed dose to primary site and CR demonstrated significant hazard ratio on OS. Multivariate analysis showed age, performance status and CR as independent predictors on two-year overall survival (Table 4). Two-year overall survival rate was statistically higher among those with complete response than those without (79.4\% [95\% CI, $67.2 \%$ to $91.6 \%$ ] vs. $33.3 \%$ [95\% CI, $7.6 \%$ to $59.9 \%$ ]; $\mathrm{p}<0.001$ ), while the median survival was 85.0 and 15.8 months $(\mathrm{p}<0.001)$ (Figure 1$)$.

\section{Failure pattern}

Of the 70 patients, isolated local-regional recurrence was observed in 10 patients (15.6\%); isolated distant metastasis was observed in seven (9.4\%); while both localregional and distant metastasis developed in 12 patients (18.8\%) (Figure 2). Of those 50 patients with CR, local recurrence occurred in 10 patients during the course of follow-up. Among whom six were muscle invasive, three were superficial, and the remaining one had missing histology record (Table 5). Among patients who developed muscle invasive recurrences, only one patient ultimately underwent radical cystectomy as salvage treatment, while the others refused salvage cystectomy or were medically

Table 4 Univariateand multivariate analysis for prognostic factors on overall survival

\begin{tabular}{|c|c|c|c|}
\hline \multicolumn{4}{|l|}{ Univariate analysis } \\
\hline & Hazard ratio & $95 \%$ confidence interval & $P$ value \\
\hline Male gender & 0.730 & $0.327-1.627$ & 0.442 \\
\hline Age, years & 1.035 & $1.001-1.069$ & 0.039 \\
\hline History of TURBT & 0.986 & $0.465-2.087$ & 0.971 \\
\hline ECOG performance status & 3.182 & $1.388-7.291$ & 0.006 \\
\hline Comorbidities & 2.264 & $0.929-5.513$ & 0.072 \\
\hline Clinical T stage & 1.565 & $1.010-2.421$ & 0.045 \\
\hline Hydronephrosis & 1.825 & $0.901-3.692$ & 0.095 \\
\hline RT duration, days & 1.016 & $0.991-1.041$ & 0.196 \\
\hline Concurrent chemotherapy & 0.587 & $0.271-1.268$ & 0.175 \\
\hline Prescribed doses to bladder tumor bed, Gy & 0.914 & $0.858-0.972$ & 0.005 \\
\hline Prescribed doses to whole bladder, Gy & 1.001 & $0.999-1.001$ & 0.245 \\
\hline Complete response (CR) & 0.155 & $0.066-0.358$ & $<0.001$ \\
\hline \multicolumn{4}{|l|}{ Multivariate analysis } \\
\hline Age & 1.064 & $1.023-1.107$ & 0.002 \\
\hline Performance status & 2.840 & $1.133-7.119$ & 0.026 \\
\hline Complete response (CR) & 0.098 & $0.040-0.240$ & $<0.001$ \\
\hline
\end{tabular}




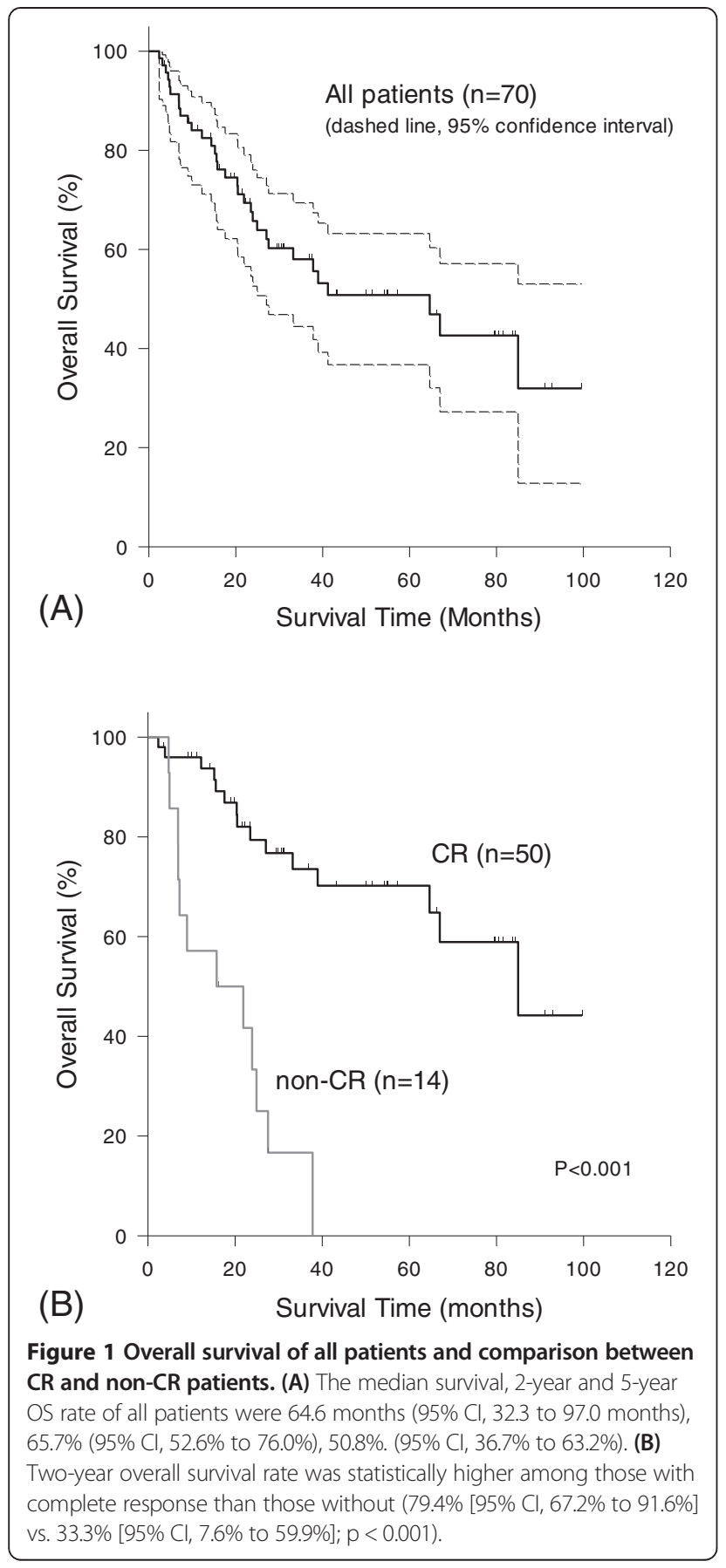

inoperable due to old age or comorbidity. None of the patients with superficial recurrences died, whereas three of the invasive recurrences did. Distant metastasis developed in one of the surviving patients with muscle invasive recurrences.

\section{Acute toxicity and chronic sequelae}

All of the radiation induced acute genitourinary (GU) and gastrointestinal (GI) toxicities and were limited to grade 2 (Table 6), and manageable. Acute grade $2 \mathrm{GU}$

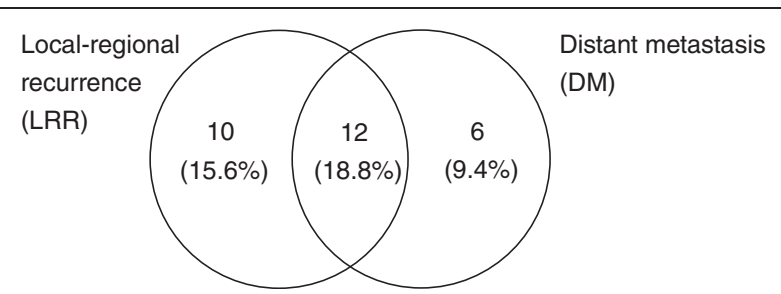

Figure 2 Failure pattern. The numbers in the circles were the patient numbers of relevant failure patterns. The percentage of relevant failure patterns were shown in the parentheses.

toxicities accounted for $28.6 \%$ and grade 2 GI toxicities in $11.4 \%$. Chronic GU and GI toxicities of grade 2 or more were encountered in 11 and 4 patients, respectively, while no patient with $\geqq$ grade $3 \mathrm{GI}$ toxicity. Chronic grade $3 \mathrm{GU}$ toxicity developed in one patient and manifested as gross hematuria and needed repeated blood transfusion. No patient died from toxicities.

\section{Discussion}

A trimodality strategy for muscle-invasive bladder cancer as the main treatment strategy for muscle-invasive bladder cancer therapy has gained its popularity over time. However, an upfront CCRT approach without NAC is relatively infrequent.

Despite the relatively low dose density of chemotherapy in CCRT phase and a low radiation dose to the whole bladder in this study, our result of a 5 year OS of $50.8 \%$ compared reasonably to most prospective trials [7,9,18-20] and cystectomy series [21,22]. A clinical CR rate of $78.1 \%$ and 5 year bladder intact survival of $49.0 \%$ in this study also compared reasonably to most upfront CCRT trials. Although NAC with platinum-based regimen before cystectomy compared to cystectomy was associated with a $5 \%$ survival benefit, the possible benefit of NAC before definitive CCRT has not been firmly established. Most NAC trials were single-armed [8,10,23,24], with one randomized trial demonstrating no statistically significant benefit [4].

Table 5 Pattern of local recurrence in CR patients $(n=10)$

\begin{tabular}{lcc}
\hline & $\begin{array}{c}\text { Patient } \\
\text { number }\end{array}$ & $\begin{array}{c}\text { Status after salvage } \\
\text { treatment }\end{array}$ \\
\hline Superficial & & \\
Papillary non-invasive carcinoma & $1(10 \%)$ & Alive with NED: 1 patient \\
Flat carcinoma in situ & $2(20 \%)$ & Alive with NED: 2 patients \\
& & Alive with NED: 2 patients \\
Invasive urothelial carcinoma & $6(60 \%)$ & Alive with distant mets: \\
& & Expired: 3 patients \\
Missing record & $1(10 \%)$ & Expired: 1 patient \\
\hline
\end{tabular}


Table 6 Toxicities

\begin{tabular}{|c|c|c|c|c|c|}
\hline & Grade 1 & Grade 2 & Grade 3 & Grade 4 & Grade 5 \\
\hline Acute GU toxicity & $21(30 \%)$ & $20(28.6 \%)$ & $0(0 \%)$ & $0(0 \%)$ & $0(0 \%)$ \\
\hline Acute Gl toxicity & $8(11.4 \%)$ & $8(11.4 \%)$ & $0(0 \%)$ & $0(0 \%)$ & $0(0 \%)$ \\
\hline Late GU toxicity & $22(31.4 \%)$ & 10 (14.3\%) & $1(1.43 \%)$ & $0(0 \%)$ & $0(0 \%)$ \\
\hline Late GI toxicity & $1(1.43 \%)$ & $4(5.7 \%)$ & $0(0 \%)$ & $0(0 \%)$ & $0(0 \%)$ \\
\hline
\end{tabular}

The presence of CR was important in predicting OS was not an exception in ours as most prospective $[4,19,25]$ and retrospective series $[5,26,27]$. In one retrospective series, CR in stage IV bladder cancer had significantly better prognosis than those with stage II or III disease but with no CR [26]. In this study, those who failed to achieve CR fared poorly without radical cystectomy. None of the non-CR patients was alive at 5 year. The normogram developed at Massechuses General Hospital (MGH) can be used to predict response to CCRT, in which hydronephrosis was one of the poor response indicators [28]. However, the presence of hydronephrosis might not be a contraindication to bladder organ preservation treatment, because a significant proportion of patients (43.3\%) in our study presented with hydronephrosis and the rate of CR was compatible to most publications. Biomarkers might be useful to better select patients for a trimodality approach and should be evaluated in between prospective trials [29].

A total of 10 local recurrences (20\%) developed among $50 \mathrm{CR}$ patients. Thirty percent of them were superficial recurrence whereas most of them were still muscleinvasive. The proportion of muscle invasive recurrences in our study seemed higher than other reports. In RTOG 95-06, the number of muscle invasive recurrence were only half of superficial recurrences [19]. Our results of a 2-year bladder intact survival rate of $64.2 \%$ compared favorably to the result of RTOG 8903 that had 99 patients without hydronephrosis [4]. In our study, the overall $20 \%$ recurrence rate in CR patients is close to that observed in the Paris group, which reported a bladder recurrence rate of $17 \%$ [30].

The optimal dose and fractionation of radiation may be important in achieving a lasting response [31]. Hyperfractionation and hypofractionation were evaluated in prospective trials [19,32]. Hypofractionated approach had better gone through whole course IMRT in order to spare normal organ at risk as much as possible. Current radiation protocol for bladder preservation regarded whole bladder dose of 54Gy and tumor bed 64-66Gy to be safe [33]. As the results of BC2001 showed no statistically increased toxicity with whole bladder irradiation to reduced high dose volume irradiation [34]. Organ motion have attributed to the uncertainties in target delineation and radiation delivery for bladder cancer treatment. Our strictly enforced bladder filling policy protocol without image-guided technique, although quite successful, should be replaced by imaged guided technique, especially for the part of tumor bed boost. The value of image guided radiotherapy (IGRT) of bladder cancer should be the mainstay of future work [35-37].

Unlike to the routine pelvic irradiation among RTOG protocols $[3,4,9,19]$, our bladder-only approach for T2 tumors and pelvic nodal coverage for T3, T4 lesions seemed appropriate. In a large retrospective series by Stein et al [38], incidence of lymph node involvement was closely related to the $\mathrm{T}$ staging of the primary bladder tumor; the incidences of lymph node involvement were $18 \%$ and $42 \%$ for T2 and T4 tumors, respectively. Our radiation protocol resulted in low locoregional failure rate $(15.6 \%)$. In additional to the low incidence of regional failure, our study also demonstrated very low incidence of late grade toxicity $(1.43 \%$ of late GU toxicity and $0 \%$ of late GI toxicity). Acute grade 3 or more toxicity was not seen. The bladder-only irradiation in combination with 3D-CRT and IMRT to T2 disease may be a sound alternative to pelvic irradiation.

The distant metastasis free survival rate in our study is $73.5 \%$ and $67.1 \%$ at 2- and 5-year, respectively. Extravesicle diseases were consistently associated with high rates of distant metastasis. The value of adjuvant chemotherapy in bladder preservation was investigated in RTOG 97-06 [32]. Although the result was promising, less than half of the enrolled patients completed the designated treatment. Adjuvant chemotherapy was not part of our treatment strategy in patients without pelvic node metastasis, even though a substantial proportion of extravesicle tumors in our study $(\mathrm{T} 3+\mathrm{T} 4=41.5 \%)$. Yet this did not jeopardize our OS rate. Unless randomized trial has proven NAC followed by CCRT and followed by chemotherapy is better CCRT alone, our upfront CCRT strategy will be continued.

\section{Conclusions}

Aim of bladder preservation therapy is to offer a quality of life treatment and avoid potential morbidity from chemotherapy or radiotherapy without compromising bladder preservation rate. Upfront CCRT with mild weekly cisplatin regimen without NAC or adjuvant chemotherapy could provide reasonable CR rate, OS and bladder preservation rate in T2-4N0 stage bladder cancer patients. 


\section{Abbreviations}

TURBT: Transurethral resection of bladder tumor; RT: Radiotherapy; CCRT: Combined chemotherapy and radiotherapy; 3D-CRT: 3D Conformal radiotherapy; IMRT: Intensity modulated radiotherapy; NAC: Neoadjuvant chemotherapy; RTOG: Radiation Therapy Oncology Group; OS: Overall survival; PFS: Progression-free survival; CR: Complete response; GU: Genitourinary; CT: Computed tomography; MRI: Magnetic resonance imaging; AJCC: American Joint Committee on Cancer; GTV: Gross tumor volume; CTV-H: Clinical target volumes at high risk; PTV-H: Planning target volume at high risk; CTV-M: Clinical target volume at intermediate risk; PTV-M: Planning target volume at intermediate risk; GFR: Glomerular filtration rate; RECIST: Response Evaluation Criteria in Solid Tumors; EORTC: European Organization for Research and Treatment of Cancer; SPSS: Statistical Package for Social Sciences software; PR: Partial response; GI: Gastrointestinal; MGH: Massechuses General Hospital; IGRT: Image guided radiotherapy.

\section{Competing interests}

The authors declare that they have no competing interests.

\section{Authors' contributions}

CYL and KLY were responsible for study design, data acquisition and manuscript preparation. KLY also performed data analysis and made all the figures \& tables. HLK, RYH, PPT, MTC, GDJ and KHC made diagnosis, collected clinicopathological data, contributed the cases and evaluated response. GDJ and $\mathrm{KHC}$ were responsible for study concept, supervision, administration support, and modifying the final manuscript. All co-authors read and approved the manuscript.

\section{Acknowledgements}

Authors would like to thank Miss Su-Chen Huang for the administrative paperwork about Institutional Review Board of our hospital.

\section{Author details}

${ }^{1}$ Department of Radiation Therapy and Oncology, Shin Kong Wu Ho-Su Memorial Hospital, No. 95, Wen-Chang Road, Shih-Lin District, Taipei City, Taiwan. ²Division of Urology, Department of Surgery, Shin Kong Wu Ho-Su Memorial Hospital, No. 95, Wen-Chang Road, Shih-Lin District, Taipei City, Taiwan. ${ }^{3}$ Shu-Tien Urology Ophthalmology Clinic, No. 276, Sec. 2, Jianguo South Road, Taipei City, Taiwan. ${ }^{4}$ School of Medicine, Fu Jen Catholic University, No. 510, Chung-Cheng Road, Hsin-Chuang, New Taipei City, Taiwan. ${ }^{5}$ School of Medicine and Institute of Biomedical Imaging and Radiological Sciences, National Yang-Ming University, No. 155, Sec. 2, Linong Street, Beitou District, Taipei City, Taiwan.

Received: 25 June 2014 Accepted: 14 September 2014

Published: 24 September 2014

\section{References}

1. Mak RH, Zietman AL, Heney NM, Kaufman DS, Shipley WU: Bladder preservation: optimizing radiotherapy and integrated treatment strategies. BJU Int 2008, 102:1345-1353.

2. Shelley MD, Barber J, Wilt T, Mason MD: Surgery versus radiotherapy for muscle invasive bladder cancer. Cochrane Database Syst Rev 2002, CD002079.

3. Tester W, Porter A, Asbell S, Coughlin C, Heaney J, Krall J, Martz K, Venner P, Hammond E: Combined modality program with possible organ preservation for invasive bladder carcinoma: results of RTOG protocol 85-12. Int J Radiat Oncol Biol Phys 1993, 25:783-790.

4. Shipley WU, Winter KA, Kaufman DS, Lee WR, Heney NM, Tester WR, Donnelly BJ, Venner PM, Perez CA, Murray KJ, Doggett RS, True LD: Phase III trial of neoadjuvant chemotherapy in patients with invasive bladder cancer treated with selective bladder preservation by combined radiation therapy and chemotherapy: initial results of Radiation Therapy Oncology Group 89-03. J Clin Oncol 1998, 16:3576-3583.

5. Rodel C, Grabenbauer GG, Kuhn R, Papadopoulos T, Dunst J, Meyer M, Schrott KM, Sauer R: Combined-modality treatment and selective organ preservation in invasive bladder cancer: long-term results. $J$ Clin Oncol 2002, 20:3061-3071.

6. James ND, Hussain SA, Hall E, Jenkins P, Tremlett J, Rawlings C, Crundwell M, Sizer B, Sreenivasan T, Hendron C, Lewis R, Waters R, Huddart RA: Radiotherapy with or without chemotherapy in muscle-invasive bladder cancer. N Engl J Med 2012, 366:1477-1488.
7. Shipley WU, Kaufman DS, Tester WJ, Pilepich MV, Sandler HM: Overview of bladder cancer trials in the Radiation Therapy Oncology Group. Cancer 2003, 97:2115-2119.

8. Dunst J, Diestelhorst A, Kuhn R, Muller AC, Scholz HJ, Fornara P: Organ-sparing treatment in muscle-invasive bladder cancer. Strahlenther Onkol 2005, 181:632-637.

9. Kaufman DS, Winter KA, Shipley WU, Heney NM, Wallace HJ 3rd, Toonkel LM, Zietman AL, Tanguay S, Sandler HM: Phase I-II RTOG study (99-06) of patients with muscle-invasive bladder cancer undergoing transurethral surgery, paclitaxel, cisplatin, and twice-daily radiotherapy followed by selective bladder preservation or radical cystectomy and adjuvant chemotherapy. Urology 2009, 73:833-837.

10. Kragelj B, Zaletel-Kragelj L, Sedmak B, Cufer T, Cervek J: Phase II study of radiochemotherapy with vinblastine in invasive bladder cancer. Radiother Oncol 2005, 75:44-47.

11. Mitin T, Hunt D, Shipley WU, Kaufman DS, Uzzo R, Wu CL, Buyyounouski MK, Sandler H, Zietman AL: Transurethral surgery and twice-daily radiation plus paclitaxel-cisplatin or fluorouracil-cisplatin with selective bladder preservation and adjuvant chemotherapy for patients with muscle invasive bladder cancer (RTOG 0233): a randomised multicentre phase 2 trial. Lancet Oncol 2013, 14:863-872.

12. Turgeon GA, Souhami L, Cury FL, Faria SL, Duclos M, Sturgeon J, Kassouf W: Hypofractionated intensity modulated radiation therapy in combined modality treatment for bladder preservation in elderly patients with invasive bladder cancer. Int J Radiat Oncol Biol Phys 2014, 88:326-331.

13. Caffo O, Fellin G, Graffer U, Luciani L: Assessment of quality of life after cystectomy or conservative therapy for patients with infiltrating bladder carcinoma. A survey by a self-administered questionnaire. Cancer 1996, 78:1089-1097.

14. Fedeli U, Fedewa SA, Ward EM: Treatment of muscle invasive bladder cancer: evidence from the National Cancer Database, 2003 to 2007. J Urol 2011, 185:72-78.

15. Eisenhauer EA, Therasse P, Bogaerts J, Schwartz LH, Sargent D, Ford R, Dancey J, Arbuck S, Gwyther S, Mooney M, Rubinstein L, Shankar L, Dodd L, Kaplan R, Lacombe D, Verweij J: New response evaluation criteria in solid tumours: revised RECIST guideline (version 1.1). Eur J Cancer 2009, 45:228-247.

16. Cox JD, Stetz J, Pajak TF: Toxicity criteria of the Radiation Therapy Oncology Group (RTOG) and the European Organization for Research and Treatment of Cancer (EORTC). Int J Radiat Oncol Biol Phys 1995, 31:1341-1346.

17. Kawashima A, Nakayama M, Kakuta Y, Abe T, Hatano K, Mukai M, Nagahara A, Nakai Y, Oka D, Takayama H, Yoshioka T, Hoshida Y, Itatani H, Nishimura $\mathrm{K}$, Nonomura N: Excision repair cross-complementing group 1 may predict the efficacy of chemoradiation therapy for muscle-invasive bladder cancer. Clin Cancer Res 2011, 17:2561-2569.

18. Efstathiou JA, Spiegel DY, Shipley WU, Heney NM, Kaufman DS, Niemierko A, Coen JJ, Skowronski RY, Paly JJ, McGovern FJ, Zietman AL: Long-term outcomes of selective bladder preservation by combined-modality therapy for invasive bladder cancer: the MGH experience. Eur Urol 2012, 61:705-711.

19. Kaufman DS, Winter KA, Shipley WU, Heney NM, Chetner MP, Souhami L, Zlotecki RA, Sause WT, True LD: The initial results in muscle-invading bladder cancer of RTOG 95-06: phase I/II trial of transurethral surgery plus radiation therapy with concurrent cisplatin and 5-fluorouracil followed by selective bladder preservation or cystectomy depending on the initial response. Oncologist 2000, 5:471-476.

20. Caffo O, Veccia A, Fellin G, Russo L, Mussari S, Galligioni E: Trimodality treatment in the conservative management of infiltrating bladder cancer: a critical review of the literature. Crit Rev Oncol Hematol 2013, 86:176-190.

21. Stein JP, Lieskovsky G, Cote R, Groshen S, Feng AC, Boyd S, Skinner E, Bochner B, Thangathurai D, Mikhail M, Raghavan D, Skinner DG: Radical cystectomy in the treatment of invasive bladder cancer: long-term results in 1,054 patients. J Clin Oncol 2001, 19:666-675.

22. Grossman HB, Natale RB, Tangen CM, Speights VO, Vogelzang NJ, Trump DL, de Vere White RW, Sarosdy MF, Wood DP Jr, Raghavan D, Crawford ED: Neoadjuvant chemotherapy plus cystectomy compared with cystectomy alone for locally advanced bladder cancer. N Engl J Med 2003, 349:859-866.

23. Gamal El-Deen H, Elshazly HF, Abo Zeina EA: Clinical experience with radiotherapy alone and radiochemotherapy with platin based regimens in organ-sparing treatment of invasive bladder cancer. J Egypt Natl Canc Inst 2009, 21:59-70. 
24. Koga F, Kihara K: Selective bladder preservation with curative intent for muscle-invasive bladder cancer: a contemporary review. Int J Urol 2012, 19:388-401.

25. Shipley WU, Kaufman DS, Zehr E, Heney NM, Lane SC, Thakral HK, Althausen AF, Zietman AL: Selective bladder preservation by combined modality protocol treatment: long-term outcomes of 190 patients with invasive bladder cancer. Urology 2002, 60:62-67. discussion 67-68.

26. Wu CE, Lin YC, Hong JH, Chuang CK, Pang ST, Liaw CC: Prognostic value of complete response in patients with muscle-invasive bladder cancer undergoing concurrent chemoradiotherapy. Anticancer Res 2013, 33:2605-2610

27. Peyromaure M, Slama J, Beuzeboc P, Ponvert D, Debre B, Zerbib M: Concurrent chemoradiotherapy for clinical stage $\mathrm{T} 2$ bladder cancer: report of a single institution. Urology 2004, 63:73-77.

28. Coen JJ, Paly JJ, Niemierko A, Kaufman DS, Heney NM, Spiegel DY, Efstathiou JA, Zietman AL, Shipley WU: Nomograms predicting response to therapy and outcomes after bladder-preserving trimodality therapy for muscle-invasive bladder cancer. Int J Radiat Oncol Biol Phys 2013, 86:311-316.

29. Catto JW, Azzouzi AR, Rehman I, Feeley KM, Cross SS, Amira N, Fromont G, Sibony M, Cussenot O, Meuth M, Hamdy FC: Promoter hypermethylation is associated with tumor location, stage, and subsequent progression in transitional cell carcinoma. J Clin Oncol 2005, 23:2903-2910.

30. Housset M: Concomitant chemoradiotherapy of infiltrating cancers of the bladder. Cancer Radiother 1998, 2:713-717.

31. Pos FJ, Hart G, Schneider C, Sminia P: Radical radiotherapy for invasive bladder cancer: What dose and fractionation schedule to choose? Int J Radiat Oncol Biol Phys 2006, 64:1168-1173.

32. Hagan MP, Winter KA, Kaufman DS, Wajsman Z, Zietman AL, Heney NM, Toonkel LM, Jones CU, Roberts JD, Shipley WU: RTOG 97-06: initial report of a phase I-II trial of selective bladder conservation using TURBT, twice-daily accelerated irradiation sensitized with cisplatin, and adjuvant MCV combination chemotherapy. Int J Radiat Oncol Biol Phys 2003, 57:665-672.

33. Ploussard G, Daneshmand S, Efstathiou JA, Herr HW, James ND, Rodel CM, Shariat SF, Shipley WU, Sternberg CN, Thalmann GN, Kassouf W: Critical analysis of bladder sparing with trimodal therapy in muscle-invasive bladder cancer: a systematic review. Eur Urol 2014, 66:120-137.

34. Huddart RA, Hall E, Hussain SA, Jenkins P, Rawlings C, Tremlett J, Crundwell M, Adab FA, Sheehan D, Syndikus I, Hendron C, Lewis R, Waters R, James ND: Randomized noninferiority trial of reduced high-dose volume versus standard volume radiation therapy for muscle-invasive bladder cancer: results of the BC2001 trial (CRUK/01/004). Int J Radiat Oncol Biol Phys 2013, 87:261-269.

35. Kron T, Wong J, Rolfo A, Pham D, Cramb J, Foroudi F: Adaptive radiotherapy for bladder cancer reduces integral dose despite daily volumetric imaging. Radiother Oncol 2010, 97:485-487.

36. Foroudi F, Wong J, Kron T, Rolfo A, Haworth A, Roxby P, Thomas J, Herschtal A, Pham D, Williams S, Tai KH, Duchesne G: Online adaptive radiotherapy for muscle-invasive bladder cancer: results of a pilot study. Int J Radiat Oncol Biol Phys 2011, 81:765-771.

37. Pos F, Bex A, Dees-Ribbers HM, Betgen A, van Herk M, Remeijer P: Lipiodol injection for target volume delineation and image guidance during radiotherapy for bladder cancer. Radiother Oncol 2009, 93:364-367.

38. Stein JP, Penson DF, Cai J, Miranda G, Skinner EC, Dunn MA, Groshen S, Lieskovsky G, Skinner DG: Radical cystectomy with extended lymphadenectomy: evaluating separate package versus en bloc submission for node positive bladder cancer. J Urol 2007, 177:876-881. discussion 881-872.

doi:10.1186/1748-717X-9-213

Cite this article as: Lee et al:: Trimodality bladder-sparing approach without neoadjuvant chemotherapy for node-negative localized muscle-invasive urinary bladder cancer resulted in comparable cystectomy-free survival. Radiation Oncology 2014 9:213.

\section{Submit your next manuscript to BioMed Central and take full advantage of:}

- Convenient online submission

- Thorough peer review

- No space constraints or color figure charges

- Immediate publication on acceptance

- Inclusion in PubMed, CAS, Scopus and Google Scholar

- Research which is freely available for redistribution

Submit your manuscript at www.biomedcentral.com/submit
C) Biomed Central 